\title{
Gastric Cancer in Pregnancy
}

\author{
ANETA TOMESCU ${ }^{1,2}$, CEZAR TOMESCU ${ }^{1,2 *}$, MARIANA ASCHIE ${ }^{1,3}$, GABRIELA STANCIU4*, RODICA SIRBU ${ }^{5}$, VLAD TICA $^{1,2}$, \\ DRAGOS BREZEANU² \\ ${ }^{1}$ Ovidius University of Constanta, Faculty of Medicine, 1 Universitatii Alley, 900470, Constanta, Romania \\ ${ }^{2}$ Second Department of Obstetrics and Gynecology, County Clinical Emergency Hospital Sf. Apostol Andrei, 145 Tomis Blvd., \\ 900591, Constanta, Romania \\ ${ }^{3}$ Anatomy and Pathology Department, County Clinical Emergency Hospital Sf. Apostol Andrei, 145 Tomis Blvd., 900591, Constanta, \\ Romania \\ ${ }^{4}$ Ovidius University of Constanta, Department of Chemistry and Chemical Engineering, 1 Universitatii Alley, 900470, Constanta, \\ Romania \\ ${ }^{5}$ Ovidius University of Constanta, Faculty of Pharmacy, 900527, Constanta, Romania
}

\begin{abstract}
Gastric cancer is an uncommon occurrence in pregnancy, but its prognosis is grim. The co-occurrence of these two symptoms makes it almost impossible for one to suspect a diagnosis of gastric cancer in a pregnant woman. Herein, we present the case of a pregnant patient, diagnosed with gastric cancer at a gestational age of 24 weeks.
\end{abstract}

Keywords: gastric cancer, pregnancy, immunohistochemistry

Gastric cancer is an uncommon occurrence in pregnancy, but its prognosis is grim. Unfortunately, some of the most frequent manifestations of this type of neoplasm include nausea and vomiting, symptoms which are also common in pregnant women [1].

Pregnancy represents a physiological stage in a women's life, which is accompanied by a plethora of endocrine and paracrine alterations. According to Uptodate.com, the incidence of gastric cancer in the United States is of approximately 22.000 new cases per year, more than half of these leading to death [2].

Pregnancy-associated gastric cancer is rare, occurring in only $0.025 \%$ to $0.1 \%$ of all pregnancies [1].

Among the main symptoms of gastric cancer we have nausea and vomiting, symptoms which commonly occur in the first trimester of pregnancy, as well as in the second or third trimester [2].

Laboratory testing includes a complete blood count, which can show an iron-deficiency anaemia, which can occur as a result of both pregnancy and gastric cancer, elevated transaminases in case of hepatic metastases, followed by imaging investigations, represented by ultrasound and upper gastrointestinal endoscopy, and, in some cases, computed tomography (CT) scan or magnetic resonance imaging (MRI) [3].

Treatment choice is based on tumour stage and consists of tumour excision, followed by chemotherapy [4].

In many cases pregnancy-associated gastric cancer is diagnosed at an advanced stage and only $45 \%$ [5] to $56 \%$ of patients undergo surgical resection [6].

\section{Experimental part}

24-year-old patient VA is admitted to the Medgidia Hospital, Obstetrics and Gynaecology Department on the May $17^{\text {th }}, 2004$ with complaints of vomiting and epigastric pain. The patient had been previously hospitalized on the April $16^{\text {th }}, 2004$ for anaemia of unknown aetiology, her CBC (complete blood count) revealing a haemoglobin level of $6.7 \mathrm{~g} / \mathrm{dL}$, for which she received two units of red blood cell concentrate.

During this admission, laboratory testing showed: Hgb $=8.9 \mathrm{~g} / \mathrm{dL}$.
During hospitalization, the patient underwent a surgical examination, during which the presence of an epigastric tumour mass was noted.

Obstetric ultrasound: monofetal pregnancy, 23-24 weeks gestational age, live foetus.

Abdominal ultrasound: liver, gallbladder, biliary tract and kidneys within normal limits. At the epigastric level, above the pancreas, a slightly hyperechoic structure with a diameter of $10 \mathrm{~cm}$, most likely belonging to the pancreas, can be observed.

The patient is transferred to the County Clinical Emergency Hospital Constanta, General Surgery $2^{\text {nd }}$ Department on the May 19 2004.

An abdominal ultrasound is performed on the May 20, 2004 which shows a visible stomach, with significantly thickened walls and inhomogeneous hyperechoic content, with a provisional diagnostic of gastric tumour mass.

On the May $21^{\text {st }}, 2004$, an obstetrical ultrasound is performed: monofetal pregnancy, of 24 weeks gestational age, live foetus.

On the May $25^{\text {th }}, 2004$, an upper Gastro- Intestinal endoscopy is performed which reveals: normal oesophagus. Normal subcardial stomach. From this level onwards a protrusive giantmass, which occupies the entire gastric body and antrum ( $>2 / 3$ of the gastric lumen), with a firm consistency and superficial ulcerations that bleed upon touch is observed. A biopsy is performed at this level. Normal D1-D2, slightly oedematous mucosa.

Conclusion: giant intragastric tumoural mass, possibly of neoplastic origin.

On the May 28 2004 , under general anaesthesia a Hoffmeister-Finsteler partial gastrectomy with gastrojejunal anastomosis is performed.

Favourable evolution in postoperative period.

The patient was discharged on the June 03, 2004 with the following diagnosis:

Serosa-invasive gastric carcinoma with multiple lymph node metastases. 24-25 weeks of pregnancy.

On the July 06, 2004, the patient is admitted to the Obstetrics and Gynaecology $1^{\text {st }}$ Department with the following diagnosis: pregnant 7 para 2, monofetal pregnancy 30-31 weeks gestational age, intact

* email: tomescu.cezar.laurentiu@gmail.com; gstanciu@univ-ovidius.ro All authors are considered main authors with equal contributions 
membranes, cephalic presentation. Operated gastric carcinoma.

On the July 07, 2004, under spinal anaesthesia and orotracheal intubation, a lower segment transverse caesarean section is performed on the patient with major maternal risk and an alive, female foetus is extracted. Weight $=1600 \mathrm{~g}$, Apgar score $=6$.

Upon extraction of the uterus, multiple small metastases, which block the uterus, intestinal loops, the omentum, and the parietal and visceral peritoneum are observed. The uterus had a friable consistency and was bleeding, which led to a drain tube being placed in the posterior vaginal culde-sac. Two blood units were transfused during the intervention.

The postoperative evolution is favourable, and an oncologic consult is requested 8 days following the intervention, with the purpose of initiating chemotherapy.

The patient is discharged on the J uly $14^{\text {th }}, 2004$, with the recommendation to return for an oncologic re-evaluation within one month.

The newborn is discharged on the August 16, 2004 with a weight of $2300 \mathrm{~g}$ and is returned to his family.

The patient dies in November 2004.

\section{Anatomic pathology}

a) Gross examination - 13/8/6 cm subtotal gastrectomy specimen: an irregular tumoural mass which occupies over $3 / 4$ of the gastric surface and ulcerates the surface epithelium; irregular surface with fatty consistency, whiteyellow in section, slightly increased consistency. Gastric slice with hyperaemic mucosa, rugal folds present. Omentum 20/10 cm with swollen capillaries and several small, white-yellowish $0.1-0.2 \mathrm{~cm}$ granulations.

Massive tumour which replaces the mucosa over more than $75 \%$ of its surface, with in-depth invasion, medium consistency and gelatinous liquid aspect in section. Tumour tissue samples, surgical slices and perigastric lymph nodes were taken and embedded in paraffin.

b) Microscopic examination - subtotal gastrectomy specimen: anaplastic carcinoma with areas of poorly differentiated mucinous and syncytial glandular carcinoma with invasion of the serosa. $2 \mathrm{~cm}$ proximal section slice with anaplastic carcinoma invasion. Distal section slice with lymphocytic infiltration and corium hyperaemia. Capillaries with carcinoma thrombi. Peritoneal fatty tissue with anaplastic carcinoma invasion. Three regional lymph nodes with a $0.5 / 0.5 \mathrm{~cm}$ diameter and massive carcinoma invasion. Fatty tissue (omentum) with anaplastic carcinoma invasion. Peritoneal metastasis.

Case re-evaluation was performed 8 years after the surgical intervention. Two paraffin blocks were analyzed, one representing tumoural tissue and the other a perigastric lymph node. The blocks were re-embedded in paraffin. 5 micron sections were created and stained with hematoxylin and eosin and periodic acid Schiff.

Slides were examines with a Leica DM750 microscope and captured with a digital Leica ICC50 HD camera. Acquisition software: LAS v4.6. Gastric specimen with massive tumor infiltration by signet ring cells and mucin

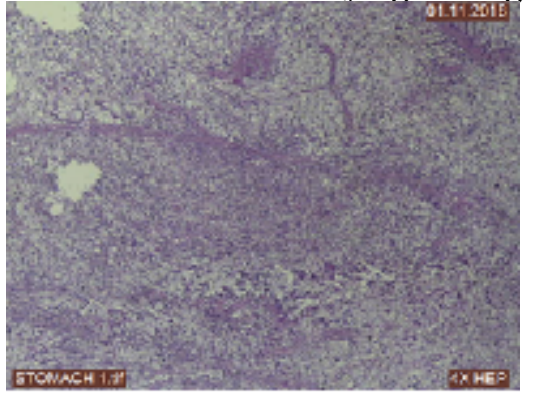

Fig. 1. Massive and diffuse tumour infiltrate

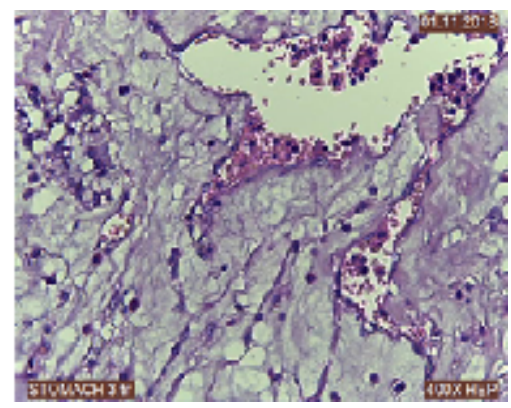

Fig. 2. Signet ring tumour cells with mucus-secreting aspect

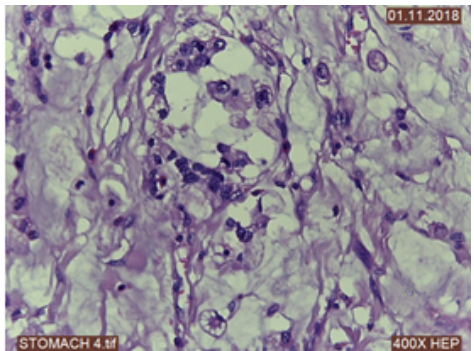

Fig. 3. Signet ring tumour cells
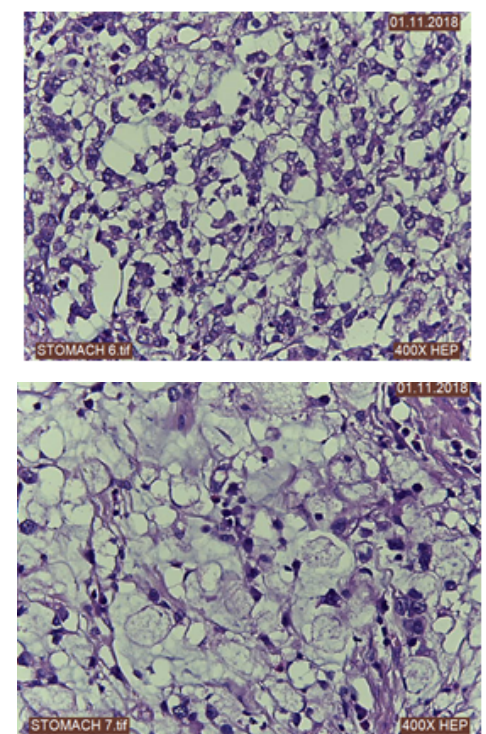

Fig. 4. Tumour and inflammatory cells

Fig 5- Tumour and inflammatory cells

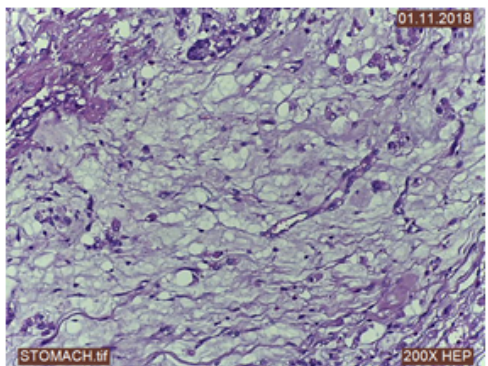

Fig 6- Increased extracellular mucin synthesis

synthesis. The gastric mucosa is completely replaced by the tumoural proliferation. PAS staining showed the presence of intra and extracellular mucin. Tumoural invasion is transmural. Perineural and vascular invasionpresent. Lymph node with tumoural invasion.

c) Immunohistochemistry was performed through the Polymer/HRP/DAB/Biocare manual method and the antibodies used were: ck7, ck20, p53. Immunohistochemistry: ck7 negative reaction, ck20 positive membrane reaction, overexpressed p53.

\section{Results and discussions}

Malignancy is unfortunately associated with pregnancy, carrying a grim prognosis for both the mother as well as for the foetus, depending on its gestational age.

The most commonly encountered malignant neoplasm in pregnancy is represented by cervical cancer, followed 
by breast cancer and melanoma. Other, less frequent malignancies are represented by ovarian cancer, lymphoma, leukaemia and colorectal cancer [7].

Pregnancy-associated gastric neoplasm is rare, with a frequency of $0.1 \%$, but it has been reported in literature. Fujimura and Fukunda described the first case of gastric cancer in a pregnant woman in 1916 [8].

Early diagnosis is difficult to establish due to the fact that it presents with symptoms common in the first trimester of pregnancy. In cases where nausea and vomiting persist beyond the $16^{\text {th }}$ week of pregnancy, a gastric malignancy should be excluded. A tardy diagnosis can lead to a spread of the malignancy throughout the entire abdomen [2].

Given the fact that gastric neoplasm often presents with epigastric complaints, its early diagnosis is cumbersome in pregnancy; consequently, early gastroscopy can be taken into account in patients with high risk [9].

Estrogenic receptors can be encountered in approximately $22 \%$ of gastric neoplastic cells, particularly in the poorly differentiated type. However, treatment using anti-oestrogen agents is controversial in both experimental studies and clinical studies [9].

In what regards pregnancy and gastrointestinal stromal tumours (GIST), immunohistochemical staining is typically positive for epidermal growth factor receptor (EGFR) and progresteron receptor (PgR) [10].

Fortunately, a gestational age of 30-31 weeks enabled foetal survival and its discharge at approximately one and a half month since birth, at a weight of $2300 \mathrm{~g}$, into its family care.

In what regards anatomic pathology and immunohistochemistry, our case presented a rare ck7-/ck20+ immune profile, suggestive for poor tumour differentiation [11-13].

p53 overexpression correlates with the aggressive character of the tumour, invasion in the gastric wall and the presence of lymph node metastasis [14].

\section{Conclusions}

The particularity of this case is represented by the presence of gastric cancer in a young, pregnant patient, without any known personal medical history at the onset of pregnancy.
Another particularity is represented by the galloping evolution of the neoplastic process in the context of pregnancy-induced immunosuppression.

The death of the patient occurred 6 months following surgical intervention for gastric cancer and 4 months following her Caesarean-section.

\section{References}

1. SAKAMOTO, K., KANDA, T., OHASHI, M., KURABAYASHI, T., SERIKAWA, T., MATSUNAGA, M., HATAKEYAMA, K., Int J Clin Oncol., 14(5), 2009, p. 392.

2. BRUGGMAN, D., BOHLMANN, M.K., BOHLE, R.M., TINNEBERG, H.R., Zentralbl Gynakol., 128(4), 2006, p. 224.

3. SIEGEL, R., MA, J., ZOU, Z., JEMAL, A., Cancer statistics, 2014. CA Cancer J Clin., 64, 9, 2014.

4. GARY, F., CUNNINGHAM, M.D., LEVENO, K. J., BLOOM, S. L., Williams Obstetrics, 24 $4^{\text {th }}$ Ed, Romanian edition, Mc Graw Hill, Sarcina si afectiunile neoplazice, 2017, p. 1219-1238,

5. CHONG, V.H., LIM, C.C., Singapore Med. J., 44(9), 2003, p. 471.

6. SILVERBERG, E. and LUBERA, J. A., Cancer statistics, 1989, Cancer Journal for Clinicians, 39(1), 1989, p. 3.

7. KIMBERLY, K. L., Cancer complicating pregnancy, Ob. Gin. Clin. N. AM. 32, $2005 X V-X V I$, preface, 2005

8. FAZENY, B., MAROSI, C., Acta Obstetricia et Gynecologica Scandinavica, 77(4), 1998, p. 469.

9. J ASPERS, V.K., GILLESSEN, A., QUAKERNACK, K., European J ournal of Obstetetrics and Gynecology and Reproductive Biology., 87(1), 1999, p. 13.

10. LANZAFAME, S., MINUTOLO, V., CALTABIANO, R., MINUTOLO, O., MARINO, B., GAGLIANO, G., D'ASTA, S., Pathol. Re.s Pract., 202(2), 2006, p. 119.

11. TAKAMI, H., SENTANI, K., MATSUDA, M., OUE, N., SAKAMOTO, N., YASUI, W., Pathobiology, 79, 2012, p. 154.

12. KRASINSKAS, A., GOLDSMITH, J., Immunohistochemistry of the Gastrointestinal Tract. Diagnostic Immunohistochemistry: Theranostic and Genomic Applications. Edited by Dabbs D. Philadelphia, PA, Saunders Elsevier, 2010, p. 505.

13. IKOMA, S., QIAO, J., RIDDLE, N.D., Austin Journal of Clinical Pathology, 2(3), 2015, 1032.

14. MALINI, K.P., SRIVANI, O., KUMAR, S., International Archives of Integrated Medicine, 3(11), 2016, p. 57.

Manuscript received:14.12.2018 